\title{
Psicoterapia psicoanalítica focalizada, implicaciones académicas en estudiantes universitarios. El modelo ESPORA ${ }^{1}$
}

\author{
Focused Psychoanalytic Psychotherapy, Academic Implications \\ in University Students. The ESPORA Model
}

Bertha Blum Grynberg $\left(^{*}\right)$ bblumg@gmail.com
Catalina Stern Forgach $\left(^{*}\right)$ catalina@ciencias.unam.mx
Dení Stincer Gómez $\left(^{*}\right)$ dstincerg@gmail.com
José Vicente Zarco Torres $\left(^{*}\right)$ vicentezarco@gmail.com
Janett Esmeralda Sosa Torralba (*) jan.estpsicol@gmail.com

(*) Universidad Nacional Autónoma de México

(Recibido: 30 de agosto de 2017; Aceptado para su publicación: 7 de febrero de 2018)

Cómo citar: Blum, B., Stern, C., Stincer, D., Zarco, J. V. y Sosa, J. E. (2019). Psicoterapia psicoanalítica focalizada, implicaciones académicas en estudiantes universitarios. El modelo ESPORA. Revista Electrónica de Investigación Educativa, 21, e40, 1-11. doi:10.24320/redie.2019.21.e40.2146

\section{Resumen}

La presente investigación tuvo el propósito de constatar estadísticamente la efectividad psicoterapéutica de un modelo de intervención focalizado y de orientación psicoanalítica (ESPORA) sobre el desempeño académico de estudiantes universitarios de la Facultad de Ciencias de la Universidad Nacional Autónoma de México. Específicamente en el análisis de sus promedios y el número de materias inscritas y aprobadas. Se realizó una comparación del comportamiento de ambas variables antes y después del tratamiento en dos grupos de estudiantes, 432 que fueron atendidos y concluyeron el tratamiento y 204 que solicitaron el servicio, pero no lo recibieron. Se aplicó el estadístico Wilcoxon-Mann-Whitney y se obtuvo que entre ambos grupos existen diferencias significativas, de lo cual se puede inferir que el modelo es eficaz y señala la necesidad de institucionalizar este tipo de modelo en espacios universitarios con el objetivo de mejorar su vida académica y evitar la deserción escolar.

Palabras clave: Efectividad psicoterapéutica, psicoterapia psicoanalítica, rendimiento académico, educación superior.

\section{Abstract}

The present investigation had the purpose to statistically verify the psychotherapeutic effectiveness of a focused intervention model and psychoanalytic orientation (ESPORA) on the academic performance of university students of the Faculty of Sciences of the UNAM. Specifically, in the analysis of their averages and the number of subjects registered and approved. A comparison was made of the behavior of both variables before and after treatment in two groups of students, 432 who were treated and completed the treatment and 204 who requested the service, but did not receive it. The Wilcoxon - Mann Whitney

\footnotetext{
${ }^{1}$ Proyecto PAPIIT IN306916.
} 
statistic was applied and it was found that there are significant differences between both groups, from which it can be inferred that the model is effective and points out the need to institutionalize this type of model in university spaces with the aim of improving their academic life and avoid dropping out of school.

Keywords: Psychotherapeutic effectiveness, psychoanalytic psychotherapy, academic performance, higher education.

\section{Introducción}

En el año 2001 la Organización Mundial de la Salud (OMS, 2001) dedicó su informe anual a la salud mental. En dicho texto se reconoce por primera vez que los factores psicológicos son fundamentales para el bienestar general de las personas, las sociedades y los países.

En el año 2011, Gore et al. (2011) hicieron especial énfasis en la salud mental de los jóvenes. En su investigación señalaron que los problemas mentales en personas de 10 a 24 años eran la principal causa de discapacidad en el mundo, siendo las problemáticas más frecuentes la depresión severa, trastorno bipolar, trastornos esquizoides y las adicciones.

Una investigación aplicada en el año 2011 a más de 57,000 estudiantes de la Universidad Nacional Autónoma de México (UNAM) arrojó que 8,197 padecían sintomatología depresiva grave (14.7\%) (González-Forteza et al., 2011); esta cifra resulta alarmante si se toman en cuenta, además, los frecuentes trastornos de ansiedad y de adicciones en estas edades (Instituto Nacional de Psiquiatría Ramón de la Fuente Muñiz/ Secretaría de Salud (2012).

Estos padecimientos psicológicos ocasionan un deterioro importante de la calidad de vida del joven, incluída el área académica: la deserción escolar, el bajo rendimiento y la desmotivación por el estudio son algunas de sus manifestaciones. Con estas condiciones corresponde asumir, por parte del sector educativo, una responsabilidad ética para atender la problemática.

En la UnAm, el Colegio de Directores de Facultades y Escuelas dio a conocer un Plan de Apoyo Integral para el Desempeño Escolar (PAIDEA). Dicho plan pretendía:

Dotar de servicios de apoyo psicológico a los alumnos, principalmente a aquellos con algún signo de desventaja que ponga en riesgo la permanencia y la conclusión de sus estudios y, de esta forma, contribuir al mejoramiento de su desempeño escolar. (PAIDEA, 2012, p. 3).

En el caso de la Facultad de Ciencias, la preocupación de las autoridades se materializó en el establecimiento de un convenio de colaboración con la Facultad de Psicología para brindar servicio de atención psicológica a la población estudiantil. En 2011 inició en la Facultad de Ciencias el Espacio de Orientación y Atención Psicológica (ESPORA). Fue el primer espacio destinado a este fin, aunque hoy existen tres más. Fueron dos las problemáticas fundamentales que motivaron la creación de este espacio: por un lado el bajo porcentaje de eficiencia terminal de los alumnos y, por el otro, los notorios problemas psicológicos y un suicidio dentro de las instalaciones.

El propósito principal de ESPORA Psicológica es abatir, en la medida de lo posible, los malestares psicológicos que aquejan a esta población y la afectación en sus vidas. Por malestar psicológico se entiende el estado anímico que provoca en el sujeto una sensación constante o frecuente de sufrimiento o angustia, que le impide atender y responder adecuadamente a los requerimientos de diferentes áreas de su vida.

Desde nuestra perspectiva, una atención psicológica sistemática in situ tendrá un impacto positivo sobre la vida personal, social y académica de los estudiantes, y más específicamente sobre sus rendimientos y responsabilidades institucionales, como el promedio y el número de materias que inscriben y logran aprobar. Luego de seis años de experiencia con el modelo ESPORA, en el área académica es importante constatar estadísticamente el impacto del mismo sobre los dos indicadores antes mencionados. 


\subsection{Fundamentos teóricos del modelo: investimento psíquico y pensamiento}

El psicoanálisis, marco teórico que fundamenta el modelo psicoterapéutico ESPORA, plantea que en los orígenes de un sujeto, de su constitución y funcionamiento psíquicos, se encuentra la relación e intercambio con el otro humano significativo, o sea, tiene sus raíces en la intersubjetividad. Intercambios que se inscriben en el psiquismo como representaciones. Es decir, todo posible conocimiento, toda objetividad, está atravesado por subjetividades (la propia y la ajena), por todo aquello que en psicoanálisis es llamado "del orden de lo afectivo, de lo libidinal" (Bleichmar, 1999).

Así, la aproximación y el entendimiento del objeto cognitivo, la apertura al mundo exterior y la constitución del psiquismo están impregnados por un investimento afectivo y por las necesidades y deseos de los sujetos involucrados. Sin interés, sin ese plus de placer, de atracción o de inclinación subjetiva (de soporte energético, libidinal), la posibilidad de conocer, de aprehender y aprender no surge o se ve interferida. El afecto constituye de este modo, a grandes rasgos, un motor u obstáculo para el desenvolvimiento de la inteligencia, la creación y los procesos intelectuales.

De acuerdo con Green (1996):

Cuando aparecen situaciones amenazantes o desestabilizadoras, (para la identidad y la autoestima) el sentido progrediente de la actividad psíquica pierde su tendencia a la resolución de situaciones conflictivas y se transforma en regrediente y repetitivo, produciendo intensas restricciones en las modalidades de productividad simbólica. (p. 38).

Dentro de ese espectro que Green llama regrediente, cabe considerar las afectaciones que distintos trastornos psicopatológicos producen en la capacidad y los procesos intelectuales y en lo que Luzuriaga (1970) denominó mecanismos de contrainteligencia (contrafuncionamiento) o la "inteligencia contra sí misma". El no aprender como proceso activo (Álvarez, Esteban y Sauvagnat, 2004; Bion, 1978; Bleichmar, 1991, 1999, 2009; Freud, 1917/1976; Klein, 1987; Laplanche, 1988; Luzuriaga, 1970; Schlemenson, 2016; Grunin, 2014).

Entre las manifestaciones de contrainteligencia se encuentran aquellas que impiden la adquisición, conservación o elaboración de conocimientos o datos de la realidad cuando los mismos son sentidos por los estudiantes como una amenaza. Amenaza por el grado de dificultad o enigma que implican, por las exigencias inherentes al nivel universitario, por cuestionar como, de entrada, su bagaje académico y capacidades intelectuales; por atacar, en consecuencia, su identidad y poner en entredicho su valoración. La respuesta es una resistencia activa, defensiva (no consciente) para atender, retener y elaborar, o sea, para pensar y aprender. Se pierde el contacto con los objetos de conocimiento y su contexto de realidad interna y externa. Así, las funciones de atención, concentración, memoria, razonamiento se ven comprometidas.

Esta modalidad de defensa se refleja con frecuencia en expresiones de distracción, apatía, desinterés, aburrimiento, sueño, "cabeza ausente y celular presente", no asistir a clases, entre otras. En estos casos el mal rendimiento académico suele convertirse en una verdadera preocupación para quien la padece (incluidos maestros y autoridades) y constituir el motivo principal o causa primaria de una demanda de atención psicológica, perturbación que puede originar, a su vez, sentimientos de tristeza, ansiedad y baja autoestima, lo cual incide negativamente en el aprendizaje y puede provocar el deseo de no continuar los estudios, convirtiéndose en causa de deserción escolar. Cada cuadro psicopatológico (inhibición, depresión, ansiedad) incide de manera diferente en las actividades escolares, sin inferir, por supuesto, una causalidad unidireccional (causa-efecto).

Cabe analizar las distintas y contradictorias aristas de la inhibición desde una limitación funcional (necesaria) del yo, de sofocamiento del inconsciente, hasta una disfunción (patológica) que rigidiza (por exceso o déficit) el funcionamiento psíquico y restringe el despliegue intelectual (Bleichmar, 2009; Freud, 1976). 
Cuando por un exceso de angustia falla la inhibición, el sujeto se siente inundado por fantasías o sensaciones displacenteras que le impiden pensar, trabajar, dirigir las actividades hacia metas previamente establecidas. En estas condiciones atender y concentrarse son tareas casi imposibles. Рara pensar resulta imprescindible inhibir (suprimir) el exceso de afecto, separarlo de la representación (Freud, 1926/1926) y desplazarlo hacia nuevos objetos del mundo y del conocimiento.

Tras una desilusión de cualquier tipo, pero significativa para el sujeto, éste disminuye su interés por las personas y por las cosas, entre ellas, las escolares. La causa puede no ser académica, pero los efectos sí. Otros estados afectivos o cognitivos-afectivos displacenteros como la ansiedad, miedo, tristeza, "dolores morales" y culpa pueden desencadenar consecuencias semejantes.

Respecto a la inhibición generalizada que caracteriza en mayor o menor medida los estados depresivos (Freud, 1976); en la depresión existe una disminución de la capacidad para pensar o concentrarse o para tomar decisiones, todos los días Asociación Americana de Psiquiatría(2014).

La pérdida de un objeto significativo (libidinalmente cargado) y la imposibilidad de realizar un deseo son la esencia y condición de la depresión, el eje alrededor del cual giran los distintos cuadros o variantes con los que se presenta. La inhibición por la pérdida de un objeto se refleja en la ausencia de motivación para acercarse o investir cualquier otro posible interés. En el deseo por el objeto perdido se centra todo el horizonte psíquico del doliente y no deja cabida para algo o alguien más. Está fijado a ese deseo que, al mismo tiempo, siente irrealizable con los consiguientes sentimientos de impotencia y desesperanza. En estas circunstancias el campo académico es simplemente una nebulosa que está allí, pero a la que no puede acercarse.

Resulta necesario, primero, enfrentar la definitividad de la pérdida, procesar el duelo, recuperar la libido comprometida para lograr investir paulatinamente las nuevas realidades (interna y externa) que involucran amigos, estudios, otros proyectos y vida. Si no se atienden ésta y demás problemáticas clínicas, obstaculizan o reducen secundariamente la capacidad de investimento libidinal del trabajo académico y de sus producciones, empobreciendo los procesos de aprendizaje y rendimiento escolar.

\subsection{El modelo ESPORA Psicológica}

ESPORA es un modelo de intervención psicoterapéutica que se apoya en el marco teórico psicoanalítico, considerando, entre otros, los conceptos planteados e incorporando los principios de la psicoterapia psicoanalítica breve focalizada (PPBF) (Braier 2006; Balint, Orstein y Balint, 1986; Fiorini 2012; Freire, 1997; Freud, 1976; Porch y Mestre, 1994).

Los objetivos del modelo son disminuir los alcances e intensidad de los conflictos psíquicos del paciente, motivo de la intervención, tales como depresión, inhibición, ansiedad, problemas de identidad y autoestima que afectan primaria o secundariamente su vida y desempeño escolar. En términos de Green (1975) y Luzuriaga (1970), el modelo busca analizar, procesar y modificar la percepción de las situaciones relacionadas con el aprendizaje que lesionan la autoestima de los pacientes y constituyen un obstáculo para el mismo. Y siguiendo a Freud (1926/1976) y a Bleichmar (2009), al incidir psicoterapéuticamente en los determinantes de inhibiciones patológicas se abre la posibilidad de investir libidinalmente nuevos objetos de conocimiento y caminos de simbolización. La focalización de los aspectos mencionados es condición de su eficacia (Fiorini, 2012), para ello se vinculan los conflictos presentes con los eventos históricos significativos que puedan influir en éstos, se esclarecen las defensas psíquicas predominantes, los afectos y vulnerabilidades asociadas (Freire, 1997). También se considera la situación personal actual, el contexto familiar, grupal y social en el que se encuentra el joven. Y, de manera importante, su inserción en la vida universitaria.

Focalizar implica, por parte del terapeuta, actividad, concentración y alternancia entre atención flotante (propia de la técnica psicoanalítica) y atención selectiva. Por lo tanto, en términos de los pacientes, se evalúan no sólo sus problemas sino sus recursos emocionales e intelectuales (las partes "sanas") de su personalidad y la motivación para el tratamiento y el cambio. 
El modelo Espora Psicológica comprende una fase diagnóstica de dos entrevistas y una fase terapéutica de 12 sesiones de 45-50 minutos cada una. Asisten alumnos de la Facultad que solicitan atención en forma personal o vía internet; la atención la brindan psicoterapeutas con adecuada formación y experiencia clínicas (grado de maestría y especialidad en Psicoterapia) en un espacio-consultorio dentro de la institución escolar.

En la fase diagnóstica se elabora una historia clínica que contempla: motivo de consulta manifiesto y latente, antecedentes inmediatos o mediatos que desencadenan la solicitud de atención, eventos históricos, personales y familiares relacionados, aspectos vulnerables y capacidades yoicas, la relación éxito-fracaso en diferentes ámbitos y la conducta e interacción del paciente con el terapeuta (y las reacciones afectivas que le genera) durante la entrevista. En función de lo anterior el terapeuta formula un diagnóstico tentativo inicial (malestar o patología predominante) desde los puntos de vista clínico y metapsicológico.

Cuando en la fase diagnóstica el psicoterapeuta detecta que por su problemática o tipo de patología (espectro psicótico o borderline, adicciones severas) el paciente no puede ser beneficiado por la pPBF se le remite y canaliza a una institución especializada. En otros casos se plantea un tratamiento combinado interdisciplinario, psicofarmacológico-psiquiátrico y psicoterapéutico dentro del modelo ESPORA.

El dispositivo metodológico y recursos técnicos utilizados en la fase terapéutica incluyen: encuadre, observación, escucha (contenido manifiesto y latente), contención (angustia y otros afectos) junto con preguntas, señalamientos, esclarecimientos, análisis de la transferencia-contratransferencia, construcción, interpretación y recapitulación al final de la intervención.

Al terminar el proceso se lleva a cabo una evaluación doble: por un lado el paciente dispone de un formato (ficha de ingreso y egreso) para evaluar los aciertos, errores y resultados del proceso psicoterapéutico y el terapeuta, por su parte, cuenta con otro formato (hoja de apoyo para el vaciado de datos) que le permite registrar los cambios durante el tratamiento para contrastarlos con los del paciente y hacer una valoración de su trabajo. La labor de los terapeutas es discutido y supervisado en juntas clínicas semanales por el equipo profesional interviniente, coordinado por dos académicos especialistas en el tema.

\subsection{Antecedentes sobre efectividad de modelos psicoterapéuticos}

Este trabajo se encuentra dentro de la línea de investigación que pretende constatar la efectividad de modelos de intervención psicoterapéutica provenientes de las distintas corrientes teóricas conocidas como "efectividad psicoterapéutica basada en evidencias". El propósito fue obtener evidencias de la efectividad de un modelo de orientación psicoanalítica sobre el desempeño académico de estudiantes universitarios.

La efectividad de los modelos de intervención psicoanalítica ha sido cuestionada sin contar con la evidencia suficiente para ello. Al respecto, Echeburúa, De Corral y Salabarrería (2010), quienes tienen una clara tendencia hacia las terapias cognitivas-conductuales, señalan: "El grado de conocimiento sobre la efectividad de las terapias dinámicas, sistémicas y existenciales-humanistas en diferentes trastornos es desconocido en muchos casos. Sería conveniente que los terapeutas e investigadores que trabajan desde estos modelos pudieran aportar datos de interés" (p. 96). Ésta es una de nuestras aspiraciones en el ámbito académico.

Existen numerosas investigaciones que constatan la efectividad de las orientaciones psicodinámicas en la práctica clínica, por ejemplo, Shedler (2010) comenta:

La magnitud del efecto de la terapia psicodinámica es tan significativa como la reportada en otras terapias que se han promovido activamente como "basadas en la evidencia". Los pacientes que reciben terapia psicodinámica logran mantener los beneficios terapéuticos y parece que siguen mejorando después de terminado el tratamiento. (p. 98). 
Otros resultados interesantes provienen de las investigaciones de Leichsenring y Leibing (2003), quienes midieron las magnitudes del efecto de las terapias cognitivos-conductuales y de la teoría psicoanalítica, esta última resultó con un valor de magnitud mayor (1.46) respecto a la primera (que tuvo valores promedio de 1.00). La teoría psicoanalítica resultó tener también un valor de mayor magnitud en el tratamiento de trastornos de personalidad. Shedler (2010) muestra empíricamente que las psicoterapias psicoanalíticas tienen un efecto más significativo en la disminución de síntomas psiquiátricos y en el funcionamiento social de los pacientes.

Respecto a los estudios sobre efectividad terapéutica, independientemente del modelo teórico del que provenga, es importante retomar lo que Romero (2008) concluye luego de un meta-análisis de los mismos. Él considera lo siguiente:

a) La psicoterapia es más efectiva que los tratamientos placebos.

b) Las diferentes psicoterapias tienen un efecto similar. No existen diferencias significativas entre las distintas psicoterapias en cuanto a grado de efectividad. En este sentido ninguna orientación ha demostrado ser claramente más efectiva que otra.

c) Algunas intervenciones del psicoterapeuta tienen efectos negativos sobre algunos pacientes.

Es una pretensión nuestra seguir proporcionando evidencias sobre la efectividad de las prácticas psicoterapéuticas.

\section{Método}

El objetivo principal de este trabajo es constatar estadísticamente que el modelo psicoterapéutico analítico focalizado ha tenido un impacto favorable sobre el desempeño académico de los estudiantes universitarios atendidos en él. A lo largo de seis años de aplicación del modelo se planteó la siguiente pregunta: ¿Existen diferencias estadísticamente significativas entre los promedios y el número de materias inscritas y aprobadas de los estudiantes que fueron atendidos por la psicoterapia psicoanalítica breve y en el de los estudiantes que solicitaron el servicio, pero que no pudieron recibir la atención?

La hipótesis de trabajo considera que sí existen diferencias estadísticamente significativas entre los promedios y el número de materias inscritas y aprobadas, antes y después del tratamiento entre los estudiantes partícipes del modelo y aquellos que lo solicitaron, pero no fueron partícipes.

Рara lograr el objetivo se utilizó un diseño pre-experimental, exploratorio y descriptivo que retoma la población atendida, a través del modelo ESPORA, de aproximadamente 1,000 estudiantes, durante seis años.

Se recolectaron los datos (promedios y número de materias inscritas y aprobadas) de los estudiantes que solicitaron el servicio, lo recibieron y lo concluyeron, y de los que solicitaron el servicio, pero no lo recibieron. Se tomaron los datos obtenidos antes del apoyo psicoterapéutico y dos semestres posterior a éste.

\subsection{Procedimiento}

Para un alfa de 0.05 y un poder de la prueba de un 0.8 según la distribución $Z$ de contraste bilateral se estimó un tamaño muestral de 361 estudiantes (casos), teniendo en cuenta la población total de estudiantes y que la investigación realizada eгa pre-experimental. Para el cálculo se utilizó el software R y su biblioteca pwr.

La muestra total de estudiantes fue de 636 estudiantes, muy superior al tamaño estimado. Esta muestra se dividió finalmente en dos grupos. El primero estuvo conformado por un total de 432 estudiantes y el segundo por 204. Del primer grupo de estudiantes (192 hombres y 240 mujeres), la distribución por 
carrera fue la siguiente: 77 estudiantes de Actuaría, 149 de Biología, 33 de Ciencias de la Computación, 33 de Ciencias de la Tierra, 3 de Físicas Bioquímica, 93 de Física y 44 de Matemática.

En el segundo grupo (86 hombres y 117 mujeres), la distribución por carrera fue: 37 estudiantes de Actuaría 37, 89 de Biología, 13 de Ciencias de la Computación, 1 de Físicas Bioquímica, 48 de Física y 15 Matemática. Estos últimos solicitaron el servicio, pero por falta de espacios y terapeutas no pudieron recibirlo. Se consideró incluir a esta población porque, al igual que el primer grupo, solicitaron atención psicológica, pero por exceso de demanda no la recibieron. De este último grupo se tomaron igualmente sus promedios y el número de materias inscritas y aprobadas antes de solicitar el tratamiento y el de los dos semestres posteriores al tiempo que duró el tratamiento psicológico.

No se excluyeron estudiantes, pues no se encontró ninguno con limitaciones sensoriales, con deterioro cognitivo ni enfermedades mentales a los que hubiera que adaptar los instrumentos de evaluación.

El modelo, tal como se mencionó, busca disminuir la intensidad de los conflictos psíquicos y de sus efectos secundarios incapacitantes, entre otros, en particular sobre el área académica. Para conocer el efecto sobre esta área se acudó al estudio del comportamiento de dos indicadores académicos 1) los promedios obtenidos en cada materia y 2) el número de materias que inscriben y aprueban los estudiantes.

Las variables independientes recogidas fueron un grupo de estudiantes que asistió a la intervención, un grupo de estudiantes que NO asistió a la intervención, materias inscritas y materias aprobadas. Las variables se recogieron en dos momentos temporales, antes de comenzar la intervención y posterior a ella.

De la información registrada en la base de datos se reporta la que resultó al restar los promedios de referencia (aquellos obtenidos antes del tratamiento) con los obtenidos dos semestres después del tratamiento. De igual forma, con el comportamiento de las materias inscritas y aprobadas antes del tratamiento y posterior a éste.

De cada variable se realizó el análisis descriptivo tomando el valor mínimo y el valor máximo, la media, la mediana, el primer y el tercer cuartil. Se obtuvo una distribución normal y se utilizó las prueba no paramétrica de Wilcoxon-Mann-Whitney que opera sobre la mediana de la diferencia con un $p<0.05$, en esta prueba se requiere que la distribución modelo sea continua y simétrica. La hipótesis nula plantea que la diferencia obtenida debe ser igual a 0 , lo que implicaría que no hay diferencia alguna y, por lo tanto, no se encuentra algún cambio significativo, versus la hipótesis alternativa que sostiene que la diferencia tiene que ser menor que 0 . Esto último implicaría que tanto el promedio como el número de materias inscritas y aprobadas sí presentan una mejora significativa después del tratamiento. Para el procesamiento estadístico de los resultados se utilizó el software estadístico libre R.

\section{Resultados}

Es imprescindible el estudio de los estadísticos descriptivos que permiten apreciar la distribución normal o no de los datos en los grupos estudiados y, por ende, la posibilidad de aplicar o no el test de WilcoxonMann-Whitney. En la tabla I se establecen los resultados descriptivos del grupo de estudiantes que asistió a la intervención. Anterior a la intervención, la media (8.023) no difiere de la mediana (8.200); posterior a la intervención, la media (8.078) tampoco difiere de la mediana (8.250). Respecto a este grupo y a las materias inscritas (ver tabla II) antes de la intervención, su media (4.910) no difiere de la mediana (5.000) o después de la intervención, su media (4.660) no difiere de la mediana (5.000). De las materias aprobadas antes de la intervención, su media (3.394) no difiere de la mediana (4.000), o después de la intervención su media (3.190) no difiere de la mediana (3.000). Esto indica que las medias no están desplazadas ni a izquierda ni a derecha, estableciendo que no existen datos anómalos ni extremos en muestras con distribuciones normales, lo que permite aplicar la prueba de Wilcoxon-Mann-Whitney. 
Tabla I. Estadísticos descriptivos obtenidos por la población de estudiantes que asistió a la intervención

\begin{tabular}{l|c|c}
\hline Estadísticos & $\begin{array}{c}\text { Antes de la } \\
\text { intervención }\end{array}$ & $\begin{array}{c}\text { Después de la } \\
\text { intervención }\end{array}$ \\
\hline Valor mínimo & 5.000 & 5.000 \\
Primer Cuartil & 7.333 & 7.333 \\
Mediana & 8.200 & 8.250 \\
Media & 8.023 & 8.078 \\
Tercer Cuartil & 9.000 & 9.000 \\
Máximo & 10.000 & 10.000 \\
\hline
\end{tabular}

Tabla II. Estadísticos descriptivos de las materias inscritas y aprobadas antes de la intervención y posterior a ella

\begin{tabular}{l|c|c|c|c}
\hline Estadísticos & $\begin{array}{c}\text { Aprobadas } \\
\text { Antes del } \\
\text { tratamiento }\end{array}$ & $\begin{array}{c}\text { Inscritas } \\
\text { antes del } \\
\text { tratamiento }\end{array}$ & $\begin{array}{c}\text { Aprobadas } \\
\text { después del } \\
\text { tratamiento }\end{array}$ & $\begin{array}{c}\text { Inscritas } \\
\text { después del } \\
\text { tratamiento }\end{array}$ \\
\hline Valor mínimo & 0.000 & 1.000 & 0.000 & 1.000 \\
Primer Cuartil & 2.000 & 4.000 & 2.000 & 4.000 \\
Mediana & 4.000 & 5.000 & 3.000 & 5.000 \\
Media & 3.394 & 4.910 & 3.190 & 4.660 \\
Tercer Cuartil & 5.000 & 6.000 & 5.000 & 6.000 \\
Máximo & 10.000 & 11.000 & 7.000 & 10.000 \\
\hline
\end{tabular}

En la tabla III se establecen los resultados descriptivos del grupo de estudiantes que NO asistió a la intervención. Anterior a la intervención, la media (7.762) no difiere de la mediana (7.833); posterior a la intervención la media (7.927) tampoco difiere de la mediana (8.166). Respecto a este grupo y a las materias inscritas (ver tabla IV) antes de la intervención, su media (4.931) no difiere de la mediana (5.000) o después de la intervención su media (4.700) no difiere de la mediana (5.000). De las materias aprobadas antes de la intervención su media (3.340) no difiere de la mediana (4.000), o después de la intervención su media (3.167) no difiere de la mediana (3.000). Lo cual indica, al igual que en la población de estudiantes que asistieron a la intervención, que las medias no están desplazadas ni a izquierda ni a derecha, estableciendo que no existen datos anómalos ni extremos en muestras con distribuciones normales, lo que permite aplicar la prueba de Wilcoxon-Mann-Whitney.

Tabla III. Estadísticos descriptivos obtenidos por los estudiantes que NO asistieron a la intervención

\begin{tabular}{l|c|c}
\hline Estadísticos & $\begin{array}{c}\text { Antes de la } \\
\text { intervención }\end{array}$ & $\begin{array}{c}\text { Después de la } \\
\text { intervención }\end{array}$ \\
\hline Valor mínimo & 5.000 & 5.000 \\
Primer Cuartil & 7.000 & 7.200 \\
Mediana & 7.833 & 8.166 \\
Media & 7.762 & 7.927 \\
Tercer Cuartil & 8.750 & 9.000 \\
Máximo & 10.000 & 10.000 \\
\hline
\end{tabular}

Tabla IV. Estadísticos descriptivos de las materias inscritas y aprobadas antes del tratamiento y posterior a él de los estudiantes que NO asistieron a la intervención.

\begin{tabular}{l|c|c|c|c}
\hline Estadísticos & $\begin{array}{c}\text { Aprobadas } \\
\text { antes del } \\
\text { tratamiento }\end{array}$ & $\begin{array}{c}\text { Inscritas } \\
\text { antes del } \\
\text { tratamiento }\end{array}$ & $\begin{array}{c}\text { Aprobadas } \\
\text { después del } \\
\text { tratamiento }\end{array}$ & $\begin{array}{c}\text { Inscritas } \\
\text { después del } \\
\text { tratamiento }\end{array}$ \\
\hline Valor mínimo & 0.000 & 2.000 & 0.000 & 1.000 \\
Primer Cuartil & 2.000 & 4.000 & 2.000 & 4.000 \\
Mediana & 4.000 & 5.000 & 3.000 & 5.000 \\
Media & 3.340 & 4.931 & 3.167 & 4.700 \\
Tercer Cuartil & 5.000 & 5.000 & 5.000 & 6.000 \\
Máximo & 7.000 & 11.000 & 7.000 & 10.000 \\
\hline
\end{tabular}


Los resultados arrojados por el estadístico de Wilcoxon indican que sí existen diferencias significativas entre los grupos estudiados respecto al comportamiento de las calificaciones y el número de materias inscritas y aprobadas. Los estudiantes que fueron atendidos muestran que sus calificaciones y el número de materias inscritas y aprobadas mejoran, respecto a los valores que tenían antes del tratamiento $\mathrm{V}=$ 22899 , con un valor $p=0.026$ (< 0.05). El grupo que solicitó el servicio pero no fue atendido muestra una mejoría, pero significativamente menor al primer grupo, en este último caso los valores fueron $\mathrm{V}=6523$, $p=0.01026(<0.05)$. Estos resultados constatan estadísticamente que la aplicación del modelo hace la diferencia entre ambos grupos.

\section{Discusión y conclusiones}

Los resultados indican que existe una mejoría significativa en el desempeño académico de los estudiantes que asistieron al proceso psicoterapéutico que se diferencia de los que no asistieron. Los indicadores académicos analizados fueron beneficiados por el modelo. Ante esto, se puede inferir que los malestares psíquicos frecuentes en la población estudiada, los cuales son esencialmente cuadros depresivos, de inhibición y de intensa ansiedad, en primer lugar, afectan y disminuyen su rendimiento académico, y en segundo lugar se constata que el modelo de atención de psicoterapia psicoanalítica breve (ESPORA) disminuye la intensidad de esta afectación.

Retomando los supuestos teóricos de partida, se puede inferir que el proceso psicoterapéutico promovió, entre otros aspectos, el levantamiento de inhibiciones patológicas que rigidizan el trabajo intelectual e interfieren con el aprendizaje. Por otra parte, la focalización y análisis de situaciones que el paciente identifica peligrosas para su integridad permitió la disminución de los excesos de angustia y demás afectos que limitan los procesos cognitivos: atención, concentración, capacidad de abstracción y productividad simbólica. Esto se pudo observar en la mejoría de las calificaciones, significativamente mayor en el grupo de estudiantes que recibió el tratamiento respecto al grupo que lo solicitó pero no lo recibió.

En términos generales el dispositivo metodológico y estrategia empleados en el proceso de intervención permiten que el estudiante analice e interprete los distintos sentidos y entrecruzamientos causales, históricos y estructurales, de los conflictos que lo afectan y su incidencia en las distintas áreas de su vida para lograr, así, su procesamiento y transformación.

Los resultados estadísticos obtenidos se constituyen, además, en una evidencia de la efectividad psicoterapéutica de un modelo de intervención psicoanalítica. Es una respuesta a la preocupación de Echeburúa et al. (2010), quienes consideran escasas las evidencias sobre la efectividad terapéutica de modelos de intervención psicoanalítica.

Los resultados permiten concluir que la implementación de una intervención psicológica dirigida a disminuir la intensidad del malestar psíquico mejora el desempeño académico de estudiantes universitarios y, en consecuencia, se puede destacar la importancia de crear espacios y programas para este tipo de atención en el ámbito universitario.

Entre las limitaciones de este estudio no se encuentra el tamaño muestral, ya que supera con creces el tamaño estimado, aunque se aplicó a una población concreta de estudiantes en la institución universitaria. Un problema fue encontrar terapeutas para el estudio, pues esto impidió que los estudiantes del segundo grupo fueran atendidos. En este sentido, son necesarios más estudios que se dirijan a otras poblaciones o instituciones para profundizar en esta área dentro del campo psicológico y con un número de terapeutas con la formación adecuada para aplicar la intervención.

\section{Agradecimientos}

Este trabajo es producto del Proyecto "Investigación e intervención psicológica institucional a estudiantes de educación superior y su repercusión en la vida académica. Un estudio de caso del Espacio de Orientación y Atención Psicológica (ESPORA) en la facultad de Ciencias, UNAM", con clave: IN306916 
financiado por el Programa de Apoyo a Proyectos de Investigación e Innovación Tecnológica (PAPIIT).

A las psicoterapeutas maestras Laura Mariana Ortiz Hoyos, Adriana Aceves Gálvez, Leticia Adriana Rivera Castañeda y al maestro Christian Flores González, quienes realizaron las intervenciones diagnósticas y psicoterapéuticas.

\section{Referencias}

Álvarez, J. M., Esteban, R. y Sauvagnat, F. (2004). Fundamentos de psicopatología psicoanalítica. Madrid: Síntesis.

Asociación Americana de Psiquiatría (2014). DSM-5. Manual diagnóstico y estadístico de los trastornos mentales (5a. ed.). Arlington: va Edición.

Balint, M., Orstein P. H. y Balint, E. (1986). Psicoterapia focal. Gedisa: Buenos Aires.

Bion, W. R. (1978). Seminarios de psicoanálisis. Buenos Aires: Paidós.

Bleichmar, H. B. (1991). La depresión. Un estudio psicoanalítico. Argentina: Nueva visión.

Bleichmar, S. (1999). Clínica psicoanalítica y neogénesis. Argentina: Amorrortu/Editores.

Bleichmar, S. (2009). Inteligencia y simbolización. Una perspectiva psicoanalítica. Buenos Aires: Paidós.

Braier, E. (2006). Psicoterapia breve de orientación psicoanalítica. Buenos Aires: Nueva Visión.

Echeburúa, E., De Corral, P. D. y Salaberría, K. (2010). Efectividad de las terapias psicológicas: Un análisis de la realidad actual. Revista de Psicopatología y Pscicología Clínica, 15(2).

doi:10.5944/rрpc.vol.15.num.2.2010.4088

Instituto Nacional de Psiquiatría Ramón de la Fuente Muñiz/ Secretaría de Salud. (2012). Encuesta Nacional de Adicciones 2011. Drogas Ilícitas. Recuperado de

http://www.conadic.salud.gob.mx/pdfs/ENA 2011 DROGAS ILICITAS .pdf

Fiorini, H. (2012). Teoría y técnica de psicoterapias. Buenos Aires: Nueva Visión.

Freire, B. (1997). Mecanismos de acción de la psicoterapia dinámica breve. Revista de la Asociación Española de Neuropsiquiatría, 17(63), 403-410.

Freud, S. (1898-1976). Sobre el mecanismo psíquico de la desmemoria (Obras completas. Vol. III). Argentina: Amorrortu.

Freud, S. (1917/1976). Duelo y melancolía (Obras completas. Vol. XIV). Argentina: Amorrortu.

Freud, S. (1925/1976). La negación (Obras completas. Vol. XIX). Argentina: Amorrortu.

Freud, S. (1926/1976). Inhibición, síntoma y angustia (Obras completas. Vol. XX). Argentina: Amorrortu.

Freud, S. (1950). Proyecto de psicología (Obras completas. Vol. I). Argentina: Amorrortu.

Freud, S. (1976). Nuevos caminos de la psicoterapia psicoanalítica (Obras completas. Vol. XVII). Argentina: Amorrortu.

Freud, S. (1976). Sobre psicoterapia (Obras completas. Vol. II). Argentina: Amorrortu. 
González-Forteza, C., Solís, C., Jiménez, A., Hernández, I., González-González, A., Juárez, F., ... y FernándezVárela, H. (2011). Confiabilidad y validez de la escala de depresión CES-D en un censo de estudiantes de nivel medio superior y superior, en la Ciudad de México. Salud Mental, 34(1), 53-59.

Gore, F. M., Bloem, P. J., Patton, G. C., Ferguson, J., Joseph, V., Coffey, C. y Mathers, C. D. (2011). Global burden of disease in young people aged 10-24 years: a systematic analysis. The Lancet, 377(9783), 20932102.

Green, A. (1975). La concepción psicoanalítica del afecto. México: Siglo XXI.

Green, A. (1996). La metapsicología revisitada. Argentina: Eudeba.

Klein, M. (1987). La importancia de la formación de símbolos en el desarrollo del yo (Obras completas. Vol. I). Buenos Aires: Paidós.

Klein, M. (1987). Una neurosis obsesiva en una niña de seis años (Obras completas. Vol. II). Buenos Aires: Paidós.

Laplanche, J. (1988). La angustia. Problemáticas I. Argentina: Amorrortu.

Leichsenring, F. y Leibing, E. (2003). The effectiveness of psychodynamic therapy and cognitive behavior therapy in the treatment of personality disorders: a meta-analysis. American Journal of Psychiatry, 160(7), 1223-1232.

Luzuriaga, I. (1970). La inteligencia contra sí misma. Buenos Aires: Psique.

Organización Mundial de la Salud. (2001). Informe sobre la salud en el mundo 2001: Salud mental: nuevos conocimientos, nuevas esperanzas. Recuperado de https://www.who.int/whr/2001/es/

Plan de Apoyo Integral para el Desempeño Escolar de los Alumnos (2012). Universidad Nacional Autónoma de México. Recuperado de http://www.paidea.unam.mx/pdf/Marco Normativo/ LINEAMIENTOS\%20GENERALES\%20DEL\%20PAIDEA.pdf

Porch, B. y Mestre, L. (1994). Psicoterapia breve y focal dentro del punto de vista psicoanalítico. En A. Davila y J. Porch (Comps.) Manual de técnicas de psicoterapia. Un enfoque psicoanalítico (pp. 471-491). Madrid: Siglo XXI.

Romero, A. F. (2008). Factores atribucionales de la efectividad psicoterapéutica. Anales de Psicología, 24(1).

Schlemenson, S. (2016). La clínica en el tratamiento psicopedagógico. Buenos Aires: Paidós.

Schlemenson, S. y Grunin, J. (2014). Adolescencia y problemas de aprendizaje. Buenos Aires: Paidós.

Shedler, J. (2010). The efficacy of psychodynamic psychotherapy. American Psychologist, 65(2), 98-109. doi:10.1037/a0018378 